\title{
Potential impact of renin-angiotensin system inhibitors and calcium channel blockers on plasma high-molecular-weight adiponectin levels in hemodialysis patients
}

\author{
Naoki Nakagawa ${ }^{1}$, Naoyuki Yao ${ }^{2}$, Tomoya Hirayama ${ }^{2}$, Mari Ishida $^{2}$, Hironori Ishida ${ }^{2}$, Atsushi Wada ${ }^{3}$, \\ Takayuki Fujino $^{1}$, Yasuaki Saijo ${ }^{4}$, Kenjiro Kikuchi ${ }^{1}$ and Naoyuki Hasebe ${ }^{1}$
}

Although metabolic syndrome confers an increased risk of cardiovascular disease in the general population, little is known about the alteration of abdominal adiposity and its association with adipocytokines in hemodialysis patients. We investigated the plasma high-molecular-weight (HMW) adiponectin level and its relationship to visceral fat area (VFA) and various markers of atherosclerosis in hemodialysis patients. In a cross-sectional study, conventional cardiovascular risk factors, plasma total and HMW adiponectin, the number of components of the metabolic syndrome and, using computed tomography, the distribution of abdominal adiposity were assessed in 144 hemodialysis patients (90 men and 54 women; mean age, 60.7 years) and 30 age- and sex-matched patients with chronic kidney disease (CKD). Plasma HMW adiponectin levels in hemodialysis patients were significantly higher than those in patients with CKD, negatively associated with VFA and serum triglycerides and positively associated with plasma total adiponectin, as well as the HMW-to-total adiponectin ratio in men and women (all $P<0.05$ ) in a simple regression analysis. In a multiple regression analysis, VFA was a significant determinant of HMW adiponectin in hemodialysis patients. Furthermore, after adjustment for classical risk factors, HMW adiponectin levels were significantly higher in patients undergoing treatment with renin-angiotensin system inhibitors or calcium channel blockers compared with patients not undergoing such treatment. This study shows that plasma HMW adiponectin levels were negatively associated with VFA and positively associated with treatment with blockade of the renin-angiotensin system and of the calcium channel. Therefore, these drugs might be effective for improving adipocytokine-related metabolic abnormalities in hemodialysis patients.

Hypertension Research (2011) 34, 592-598; doi:10.1038/hr.2010.282; published online 3 February 2011

Keywords: calcium channel blockers; hemodialysis patients; high-molecular-weight adiponectin; renin-angiotensin system inhibitors; visceral obesity

\section{INTRODUCTION}

It is well known that metabolic disturbances in chronic kidney disease (CKD), such as insulin resistance, inflammation and lipid abnormalities, are causally associated with atherosclerosis and cardiovascular death in hemodialysis patients. ${ }^{1}$ In such patients, the risk of cardiovascular death is more than 10 times higher than that in subjects with normal kidney function. ${ }^{2}$

Adipose tissue is currently considered to be not only a reservoir for storage of energy, but also an active endocrine organ producing several adipocytokines. ${ }^{3}$ Adiponectin, a 30-kDa collagen-like protein synthesized by adipocytes, is a multifunctional adipocytokine with favorable effects on glucose and lipid metabolism, insulin resistance and inflammation, and it has been shown to play a protective role in experimental models of vascular injury. Clinically, serum or plasma adiponectin is decreased in pathological states, including obesity, diabetes mellitus and ischemic heart disease. ${ }^{4,5}$ Hypoadiponectinemia increases the prevalence of ischemic heart disease twofold ${ }^{4,5}$ and has been shown to be a risk factor for ischemic heart disease in the general population. In hemodialysis patients with cardiovascular events, serum adiponectin levels are lower than that in patients without cardiovascular events. ${ }^{6}$ Therefore, the circulating adiponectin level is regarded as an inverse predictor of cardiovascular outcome in hemodialysis patients as in the general population. Adiponectin exists in several isoforms in the human blood, including low-, middle-, and

${ }^{1}$ Department of Internal Medicine, Division of Cardiology, Nephrology, Pulmonology and Neurology, Asahikawa, Japan; ${ }^{2}$ Departments of Nephrology and Urology, Kitasaito Hospital, Asahikawa, Japan; ${ }^{3}$ Department of Nephrology, Asahikawa Red Cross Hospital, Asahikawa, Japan and ${ }^{4}$ Department of Health Science, Asahikawa Medical University, Asahikawa, Japan

Correspondence: Dr N Nakagawa, Department of Internal Medicine, Division of Cardiology, Nephrology, Pulmonology and Neurology, Asahikawa Medical University, Asahikawa 078-8510, Japan.

E-mail: naka-nao@asahikawa-med.ac.jp

Received 29 October 2010; accepted 21 November 2010; published online 3 February 2011 
high-molecular-weight (HMW) isoforms. ${ }^{7}$ The HMW form is most active and confers a protective effect on blood vessels. However, the prevalence of visceral fat obesity and the alteration of adipocytokines, such as plasma total and HMW adiponectin, tumor necrosis factor- $\alpha$ and inflammatory markers in hemodialysis patients, has not been fully elucidated. In this study, we investigated plasma HMW adiponectin levels and its relationship with various markers of atherosclerosis including visceral fat obesity, which was determined by abdominal computed tomography in Japanese hemodialysis patients. Furthermore, we investigated the effect of antiatherosclerotic treatment on HMW adiponectin and the HMW-to-total adiponectin ratio.

\section{METHODS}

\section{Subjects}

A total of 218 maintenance hemodialysis patients with standard bicarbonate dialysate, age $\geqslant 20$ years and a hemodialysis duration of $\geqslant 90$ days were enrolled. The hemodialysis dose was checked using the following formula: $K t / V=-\ln (R-0.03)+[(4-3.5 R) \times(\mathrm{UF} / W)]$, where $K t / V$ is a single-pool $K t / V$, $R$ is the ratio of post-dialysis to pre-dialysis serum urea nitrogen, $t$ is the duration of dialysis in hours, UF is the ultrafiltration volume in liters and $W$ is the post-dialysis body weight in kilograms. ${ }^{8}$ Age, gender, lipid parameters and conventional cardiovascular risk factors were also recorded. Information with regard to antihypertensive regimens, treatment with renin-angiotensin system (RAS) inhibitors, such as angiotensin-converting enzyme inhibitors and/or angiotensin II receptor blockers, calcium channel blockers (CCBs), $\beta$-blockers, statins and antidiabetic regimens, or insulin was collected. The exclusion criteria were a weekly dialysis time of $<12 \mathrm{~h}$, urea $\mathrm{Kt} / \mathrm{V}$ of $<1.2$, the use of a temporary hemodialysis catheter and comorbidity with malignancy, inflammation, infectious diseases or polycystic kidney disease. After removing these patients, we recruited 144 maintenance hemodialysis patients (90 men and 54 women; age, $60.7 \pm 11.8$ years; duration of hemodialysis, $150.4 \pm 108.7$ months) and 30 age- and sex-matched patients with CKD (18 men and 12 women; age, $61.9 \pm 12.7$ years).

This study was approved by the ethics committee of Asahikawa Medical College. Informed consent was obtained from each patient before entry into the study.

\section{Data collection}

Blood samples were taken immediately before the first dialysis session of the week in a supine position and collected in vacuum plastic tubes. The samples were centrifuged at 3000 r.p.m. for $15 \mathrm{~min}$ at $4{ }^{\circ} \mathrm{C}$. The supernatant was decanted and frozen at $-80^{\circ} \mathrm{C}$ until assayed. Plasma levels of total and HMW adiponectin and of tumor necrosis factor- $\alpha$ were measured using commercially available ELISA kits (Sekisui Medical, Tokyo, Japan and R\&D Systems, Abingdon, UK, respectively), and the HMW-to-total adiponectin ratio was calculated. Among volunteers ( 20 females and 27 males), the means of the total and HMW adiponectin levels were significantly higher in women than that in men $\left(6.62 \pm 3.04\right.$ vs. $4.30 \pm 1.76 \mu \mathrm{g} \mathrm{ml}^{-1}, 3.24 \pm 2.13$ vs. $1.62 \pm 1.02 \mu \mathrm{g} \mathrm{ml}^{-1}$, respectively; $P<0.005$, by Student's $t$-test) as described previously. ${ }^{9}$ Other parameters were measured by standard laboratory methods.

Waist circumference (WC) (in $\mathrm{cm}$ ) at the umbilical level was measured in a standing position at the start of dialysis. Body weight denotes dry weight, and body mass index (BMI) was calculated by dividing dry weight $(\mathrm{kg})$ by squared height $\left(\mathrm{m}^{2}\right)$. Smoking was defined as current smoking or a history of habitual smoking. Hypertension was defined as either systolic pressure $\geqq 140$ and/or diastolic pressure $\geqq 90 \mathrm{~mm} \mathrm{Hg}$ or current use of antihypertensive medications. Diabetes was defined as one of the following: fasting blood sugar $\geqq 126 \mathrm{mg} \mathrm{dl}^{-1}$; non-fasting blood sugar $\geqq 200 \mathrm{mg} \mathrm{dl}^{-1}$ or hemoglobin AlC $\left(\mathrm{HbA}_{1 \mathrm{c}}\right) \geqq 6.0 \%$; or current use of insulin or an oral hypoglycemic agent. Dyslipidemia was defined as: total cholesterol $\geqq 220 \mathrm{mg} \mathrm{dl}^{-1}$; high-density lipoprotein (HDL) cholesterol $<40 \mathrm{mg} \mathrm{dl}^{-1}$ for men, $<50 \mathrm{mg} \mathrm{dl}^{-1}$ for women or triglyceride $\geqq 150 \mathrm{mg} \mathrm{dl}^{-1}$; or current use of an antihyperlipidemic medication. Metabolic syndrome (MS) criteria were determined using a modified version of the National Cholesterol Education Program Expert Panel on Detection, Evaluation and Treatment of High Blood Pressure (Adult Treatment Panel III) ${ }^{10}$ as follows: (1) medication for hypertension or systolic blood pressure $(\mathrm{BP}) \geqslant 130$ and/or diastolic pressure $\geqslant 85 \mathrm{~mm} \mathrm{Hg}$; (2) triglycerides $\geqslant 150 \mathrm{mg} \mathrm{dl}^{-1}$; (3) $\mathrm{HDL}$ cholesterol $<40 \mathrm{mg} \mathrm{dl}^{-1}$ for men and $<50 \mathrm{mg} \mathrm{dl}^{-1}$ for women; (4) medication for diabetes or $\mathrm{HbA}_{\mathrm{lc}}$ $\geqslant 6.0 \%$; and (5) WC $>85 \mathrm{~cm}$ for men and $\geqslant 90 \mathrm{~cm}$ for women, which is the definition of MS in Japan. ${ }^{11}$ Because data on fasting glucose was only available in a subsample of fasting subjects, we assessed $\mathrm{HbA}_{1 \mathrm{c}}$ instead.

Abdominal fat distribution was determined using an abdominal computed tomography at the level of the umbilicus. Subcutaneous fat tissue was defined as the extraperitoneal fat between the skin and muscle. The intra-abdominal tissue with the same density as the subcutaneous fat tissue was defined as visceral fat tissue. The subcutaneous fat area and visceral fat area (VFA) were also measured at the level of the umbilicus. We investigated the prevalence of visceral fat obesity defined as VFA $>100 \mathrm{~cm}^{2}$, which is a risk factor for MS in Japan and is a visceral fat mass that is approximated by a WC of $85 \mathrm{~cm}$ in men and $90 \mathrm{~cm}$ in women. ${ }^{11}$

\section{Statistical analysis}

The results were expressed as the mean \pm s.d. or s.e.m. Univariate and multivariate linear regression was used for continuous variables. The means of HMW adiponectin and VFA were compared with the different metabolic factors with a one-way analysis of variance followed by multiple comparisons using Turkey's Studentized Range Test. The adjusted mean of HMW adiponectin was compared among the different medications with analysis covariance for age, sex, systolic BP, BMI, Hb, albumin, previous cardiovascular disease (CVD), smoking, medication for hypertension, medication for diabetes and medication for dyslipidemia. $P$-values $<0.05$ were considered statistically significant. All statistical analyses were performed with the SPSS software package version 11.0 for Windows (SPSS, Chicago, IL, USA).

\section{RESULTS}

\section{Relationship between HMW adiponectin and VFA}

The baseline characteristics of the subjects are outlined in Table 1. The prevalence of hypertension, diabetes mellitus and dyslipidemia was 81, 31 and $63 \%$, respectively, in hemodialysis patients. Table 2 shows the body composition, fat distribution and adipocytokines according to sex. Although mean BMI, WC and VFA were greater in men, no significant difference was found in subcutaneous fat area between the sexes in patients with CKD and on hemodialysis. The prevalence of VFA $>100 \mathrm{~cm}^{2}$ was $31.2 \%$ in men and $15.5 \%$ in women. There were no significant differences in age, WC, VFA or subcutaneous fat area between patients with CKD and those on hemodialysis within the same gender groups. Despite the higher body weight and BMI in female patients with CKD than those with hemodialysis, the HMW adiponectin levels were significantly higher in hemodialysis patients compared with patients with CKD in both men and women. Furthermore, HMW adiponectin levels of patients with CKD and hemodialysis were higher than those of healthy volunteers, as reported previously, ${ }^{9}$ although we did not have data from age- and sex-matched healthy volunteers.

As shown in Figure 1, a similar negative relationship between total or HMW adiponectin and VFA was observed in both men and women who were hemodialysis patients. Table 3 lists univariate correlations between HMW adiponectin and various parameters among hemodialysis patients. HMW adiponectin correlated significantly and positively with total adiponectin, HMW-to-total adiponectin ratio and HDL-C and negatively with VFA, dyslipidemia and triglycerides in both men and women who were hemodialysis patients. HMW adiponectin correlated significantly and negatively with BMI and WC in men, but not in women, suggesting that VFA is a more sensitive determinant for reduced plasma HMW adiponectin than BMI and WC in hemodialysis patients. To determine factors contributing to HMW adiponectin, we performed a multiple regression analysis for HMW adiponectin and showed that VFA was a significant determinant for 


\begin{tabular}{|c|c|c|c|c|}
\hline & \multicolumn{2}{|c|}{$C K D$} & \multicolumn{2}{|c|}{$H D$} \\
\hline & Men & Women & Men & Women \\
\hline Age (years) & $62.7 \pm 15.4$ & $61.8 \pm 8.6$ & $61.3 \pm 10.9$ & $59.7 \pm 13.0$ \\
\hline Duration of dialysis (mean) & - & - & $133.8 \pm 107.5$ & $178.6 \pm 105.8$ \\
\hline Hypertension & $13(81 \%)$ & $10(83 \%)$ & $74(82 \%)$ & $42(78 \%)$ \\
\hline Smoking & $8(50 \%)$ & $3(25 \%)$ & $40(44 \%)$ & $12(22 \%)$ \\
\hline Previous CVD & $6(38 \%)$ & $3(25 \%)$ & $25(28 \%)$ & $12(22 \%)$ \\
\hline Systolic BP (mm Hg) & $140 \pm 19$ & $138 \pm 20$ & $148 \pm 23$ & $145 \pm 19$ \\
\hline Diastolic BP (mm Hg) & $78 \pm 15$ & $77 \pm 14$ & $84 \pm 16$ & $82 \pm 13$ \\
\hline Hemoglobin $\left(\mathrm{g} \mathrm{dl}^{-1}\right)$ & $12.3 \pm 2.0$ & $10.6 \pm 1.5$ & $10.9 \pm 1.3$ & $10.4 \pm 1.0$ \\
\hline Hematocrit (\%) & $36.1 \pm 4.9$ & $32.2 \pm 3.6$ & $32.0 \pm 3.7$ & $30.8 \pm 2.8$ \\
\hline Serum uric acid $\left(\mathrm{mgdl}^{-1}\right)$ & $7.4 \pm 1.3$ & $6.9 \pm 1.9$ & $6.9 \pm 1.3$ & $7.4 \pm 1.4$ \\
\hline Serum potassium $\left(\mathrm{mEq} \mathrm{I}^{-1}\right)$ & $4.2 \pm 0.4$ & $4.4 \pm 0.3$ & $4.9 \pm 0.7$ & $4.9 \pm 0.6$ \\
\hline Serum calcium $\left(\mathrm{mgdl}^{-1}\right)$ & $9.1 \pm 0.4$ & $8.8 \pm 0.6$ & $8.9 \pm 0.9$ & $9.2 \pm 0.8$ \\
\hline Serum phosphate $\left(\mathrm{mg} \mathrm{dl}^{-1}\right)$ & $3.3 \pm 0.6$ & $4.1 \pm 0.9$ & $6.1 \pm 1.6$ & $5.9 \pm 1.9$ \\
\hline Serum intact PTH $\left(\mathrm{pg} \mathrm{ml}^{-1}\right)$ & $50.3 \pm 31.3$ & $78.5 \pm 49.3$ & $148.6 \pm 143.4$ & $136.0 \pm 148.1$ \\
\hline Serum albumin $\left(\mathrm{g} \mathrm{dl}^{-1}\right)$ & $4.0 \pm 0.5$ & $3.7 \pm 0.4$ & $3.9 \pm 0.3$ & $3.9 \pm 0.3$ \\
\hline Serum urea $\left(\mathrm{mg} \mathrm{dl}^{-1}\right)$ & $28.6 \pm 12.9$ & $37.6 \pm 19.6$ & $64.4 \pm 16.0$ & $61.8 \pm 17.3$ \\
\hline Serum creatinine $\left(\mathrm{mg} \mathrm{dl}^{-1}\right)$ & $1.6 \pm 0.8$ & $2.5 \pm 1.7$ & $12.1 \pm 3.0$ & $10.2 \pm 2.2$ \\
\hline Hemoglobin Alc (\%) & $5.6 \pm 1.1$ & $5.9 \pm 1.2$ & $5.4 \pm 0.6$ & $5.6 \pm 1.0$ \\
\hline$K t / V$ urea & - & - & $1.33 \pm 0.21$ & $1.55 \pm 0.20$ \\
\hline
\end{tabular}

Abbreviations: BP, blood pressure; CKD, chronic kidney disease; CVD, cardiovascular disease; HD, hemodialysis; HDL, high-density lipoprotein; $K t / V$, fractional urea clearance; LDL, low-density lipoprotein; PTH, parathyroid hormone.

Variables are presented as mean \pm s.d. or number (percentage).

Table 2 Body composition, fat accumulation and adipocytokines according to sex

\begin{tabular}{|c|c|c|c|c|}
\hline & \multicolumn{2}{|c|}{$C K D$} & \multicolumn{2}{|c|}{$H D$} \\
\hline & Men & Women & Men & Women \\
\hline Number & 18 & 12 & 90 & 54 \\
\hline Body weight/dry weight (kg) & $61.4 \pm 10.2$ & $57.3 \pm 9.6$ & $60.5 \pm 8.3$ & $47.5 \pm 7.1^{*, \dagger}$ \\
\hline Body mass index $\left(\mathrm{kg} \mathrm{m}^{-2}\right)$ & $22.7 \pm 2.9$ & $24.1 \pm 3.6$ & $22.4 \pm 2.6$ & $20.8 \pm 3.2^{*, \dagger}$ \\
\hline Waist circumference $(\mathrm{cm})$ & $83.3 \pm 6.7$ & $86.3 \pm 11.2$ & $85.4 \pm 8.7$ & $80.5 \pm 9.6$ \\
\hline Subcutaneous fat area $\left(\mathrm{cm}^{2}\right)$ & $90.1 \pm 33.7$ & $127.9 \pm 56.5^{*}$ & $90.3 \pm 47.9$ & $96.4 \pm 49.0$ \\
\hline Visceral fat area $\left(\mathrm{cm}^{2}\right)$ & $80.9 \pm 42.4$ & $62.3 \pm 22.9$ & $82.8 \pm 48.8$ & $57.5 \pm 36.1$ \\
\hline Visceral fat area $\geqslant 100 \mathrm{~cm}^{2}(\%)$ & 31.3 & 0.0 & 31.2 & 15.5 \\
\hline Plasma total adiponectin ( $\mu \mathrm{g} \mathrm{ml}^{-1}$ ) & $6.4 \pm 3.5$ & $9.0 \pm 6.1$ & $9.5 \pm 4.5^{\dagger}$ & $10.9 \pm 5.1$ \\
\hline Plasma HMW adiponectin ( $\left.\mu \mathrm{g} \mathrm{ml}^{-1}\right)$ & $2.3 \pm 2.0$ & $3.9 \pm 3.0$ & $4.1 \pm 2.7^{\dagger}$ & $5.3 \pm 3.4^{*, \dagger}$ \\
\hline Plasma TNF- $\alpha\left(\mathrm{pg} \mathrm{ml}^{-1}\right)$ & $1.8 \pm 1.5$ & $2.7 \pm 1.6$ & $4.2 \pm 1.7^{\dagger}$ & $4.4 \pm 1.6^{\dagger}$ \\
\hline Serum hsCRP (mg dl ${ }^{-1}$ ) & $0.22 \pm 0.33$ & $0.40 \pm 0.48$ & $0.30 \pm 0.48$ & $0.23 \pm 0.46$ \\
\hline
\end{tabular}

Abbreviations: CKD, chronic kidney disease; HD, hemodialysis; HMW, high molecular weight; TNF, tumor necrosis factor; hsCRP, high-sensitivity C-reactive protein. Variables are presented as mean \pm s.d. or percentage.

${ }^{*} P<0.05$ vs. men of same group and ${ }^{*} P<0.05$ vs. same sex of CKD.

HMW adiponectin $(r=-0.367, P<0.0001)$ in hemodialysis patients (Table 4).

\section{Relationship between MS components and HMW adiponectin or} VFA in hemodialysis patients

Figure 2 shows the relationship between the number of components of MS and HMW adiponectin or VFA. There was a stepwise decrease in
HMW adiponectin levels corresponding to the accumulation of components of MS $(P<0.0001$ for trend) (Figure $2 \mathrm{a})$. HMW adiponectin levels of subjects with three or more components of MS were significantly lower than those with none or only one component in men, but not in women because of the lower levels of HMW adiponectin in men than women with the same number of components of MS. In contrast, there was a stepwise increase in VFA 

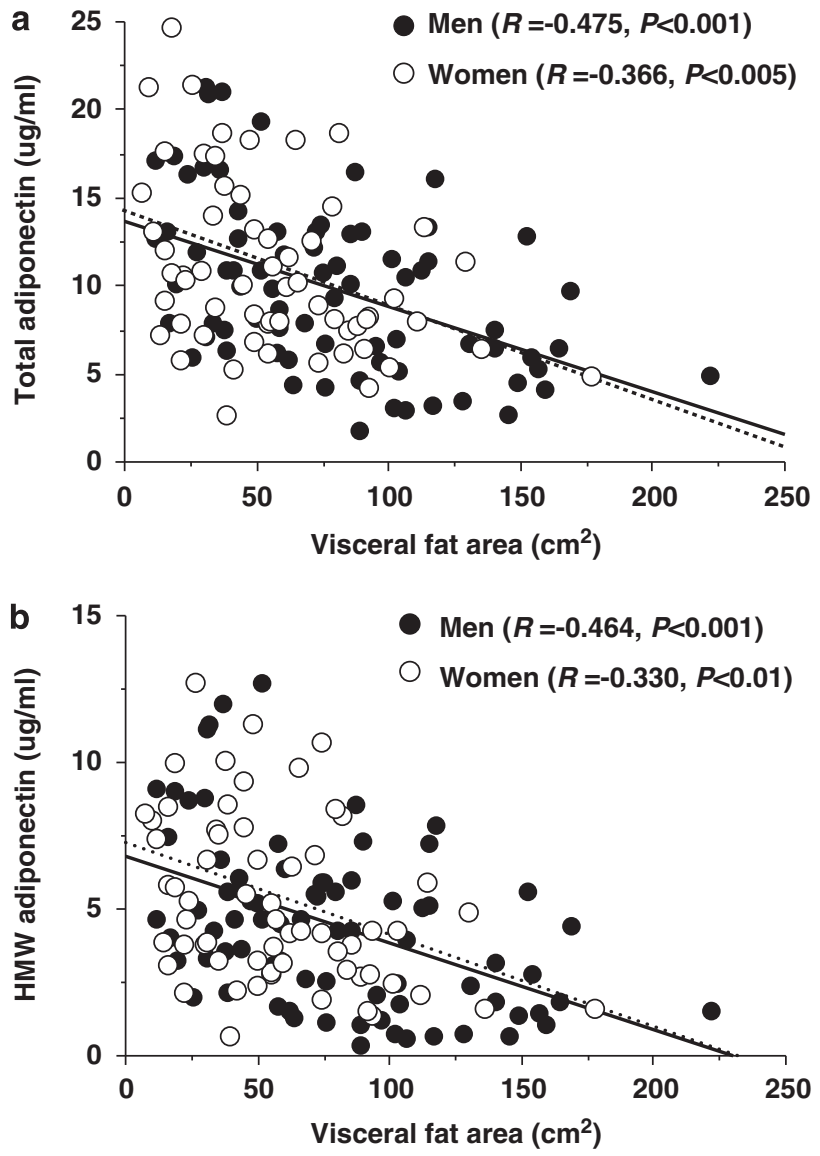

Figure 1 The relationship between the visceral fat area and plasma total adiponectin (a) or high-molecular-weight (HMW) adiponectin (b) in 90 male (filled circle; solid lines) and 54 female (open circle; dotted lines) hemodialysis patients.

corresponding to the accumulation of the components of MS $(P<0.0001$ for trend) (Figure 2b). The VFA of the subjects with three or more components of MS was significantly greater than those with none or only one component in both men and women. Furthermore, a significant difference in VFA between subjects with two and four more components were also found in both men and women, suggesting that abdominal visceral fat obesity is associated with the accumulation of MS components in hemodialysis patients as it is in non-hemodialysis patients.

Impact of RAS inhibitors and CCBs on HMW adiponectin

Finally, we investigated the relationship between HMW adiponectin or HMW-to-total adiponectin ratio and antiatherosclerotic treatments in hemodialysis patients. Figure 3 shows the differences in the HMW adiponectin and HMW-to-total adiponectin ratio according to medical treatment after adjustment for age, sex, systolic BP, BMI, Hb, albumin, previous CVD, smoking, duration of hemodialysis, medication for hypertension, medication for diabetes and medication for dyslipidemia. We found significant differences in the HMW adiponectin levels and HMW-to-total adiponectin ratio compared with patients not taking any medications; the values were greater in patients using RAS inhibitors, CCBs and a combination of RAS inhibitors and CCBs, but not in patients using $\beta$-blockers and statins. There were no significant differences in age, sex, VFA, BMI and systolic BP in patients treated with or without the use of RAS inhibitors/CCBs (Table 5),
Table 3 Univariate correlation between plasma HMW adiponectin and other parameters in hemodialysis patients

\begin{tabular}{|c|c|c|}
\hline & Men & Women \\
\hline Age & 0.128 & -0.080 \\
\hline Duration of dialysis & $0.233^{*}$ & 0.119 \\
\hline Body mass index & $-0.346^{* *}$ & -0.126 \\
\hline Waist circumference & $-0.362^{* * *}$ & -0.0091 \\
\hline Visceral fat area & $-0.487^{* * *}$ & $-0.311^{*}$ \\
\hline Subcutaneous fat area & $-0.286^{* *}$ & -0.154 \\
\hline Plasma total adiponectin & $0.951^{* * *}$ & $0.945^{* * *}$ \\
\hline HMW-to-total ratio & $0.833^{* * *}$ & $0.758^{* * *}$ \\
\hline Plasma TNF- $\alpha$ & 0.182 & 0.265 \\
\hline Serum hsCRP & 0.018 & 0.098 \\
\hline Hypertension & 0.064 & 0.105 \\
\hline Diabetes & $-0.240^{*}$ & 0.048 \\
\hline Dyslipidemia & $-0.347^{* * *}$ & $-0.388^{* *}$ \\
\hline Smoking & -0.080 & -0.010 \\
\hline Previous CVD & 0.116 & -0.019 \\
\hline Systolic BP & 0.047 & -0.041 \\
\hline Pulse pressure & 0.039 & -0.149 \\
\hline Hemoglobin & -0.065 & 0.070 \\
\hline Hematocrit & 0.002 & 0.066 \\
\hline Serum total cholesterol & 0.001 & -0.055 \\
\hline Serum triglycerides & $-0.355^{* * *}$ & $-0.303^{*}$ \\
\hline Serum HDL-cholesterol & $0.225^{*}$ & $0.259 *$ \\
\hline Serum LDL-cholesterol & 0.086 & -0.052 \\
\hline Serum uric acid & $-0.280^{* *}$ & -0.029 \\
\hline Serum potassium & 0.134 & \\
\hline Serum calcium & 0.121 & 0.240 \\
\hline Serum phosphate & 0.097 & -0.047 \\
\hline Serum intact PTH & 0.134 & -0.037 \\
\hline Serum albumin & -0.068 & 0.037 \\
\hline Serum urea & -0.073 & -0.162 \\
\hline Serum creatinine & -0.206 & -0.253 \\
\hline Hemoglobin A1C & -0.145 & -0.186 \\
\hline$K t / V$ urea & 0.046 & 0.278 \\
\hline
\end{tabular}

Abbreviations: BP, blood pressure; CVD, cardiovascular disease; HDL, high-density lipoprotein; $\mathrm{HMW}$, high molecular weight; hsCRP, high-sensitivity C-reactive protein; $K t / V$, fractional urea clearance; LDL, low-density lipoprotein; PTH, parathyroid hormone; TNF, tumor necrosis factor. ${ }^{*} P<0.05 ;{ }^{*} P<0.01 ;{ }^{* *} P<0.001$

Table 4 Multiple regression analysis of the correlation of HMW adiponectin in hemodialysis patients

\begin{tabular}{lrrrc}
\hline & $\beta$ & B & 95\% Cl & P-value \\
\hline Age & 0.108 & 0.027 & -0.017 to 0.071 & 0.229 \\
Sex (man=1, woman=0) & -0.146 & -0.891 & -1.930 to 0.149 & 0.092 \\
Duration of dialysis & 0.161 & 0.004 & -0.001 to 0.009 & 0.083 \\
BMI & 0.098 & 0.100 & -0.105 to 0.304 & 0.336 \\
Visceral fat area & -0.367 & -0.024 & -0.036 to -0.011 & $<0.0001$ \\
Hypertension & 0.052 & 0.628 & -1.222 to 2.479 & 0.503 \\
Diabetes & -0.056 & -0.368 & -1.461 to 0.725 & 0.507 \\
Dyslipidemia & -0.295 & -1.829 & -2.836 to -0.818 & 0.082 \\
Smoking & 0.103 & 0.297 & -0.214 to 0.807 & 0.253 \\
Previous CVD & 0.040 & 0.270 & -0.797 to 1.336 & 0.618 \\
Hemoglobin & -0.015 & -0.037 & -0.445 to 0.371 & 0.859 \\
Serum albumin & 0.033 & 0.335 & -1.418 to 2.087 & 0.706 \\
Serum uric acid & -0.118 & -0.258 & -0.613 to 0.096 & 0.151 \\
\hline
\end{tabular}

Abbreviations: $\beta$, standardized coefficients; $B$, unstandardized coefficients; BMI, body mass index; $\mathrm{Cl}$, confidence interval; $\mathrm{CVD}$, cardiovascular disease; $\mathrm{HMW}$, high molecular weight. Model $R^{2}=0.310 ; P<0.0001$. 

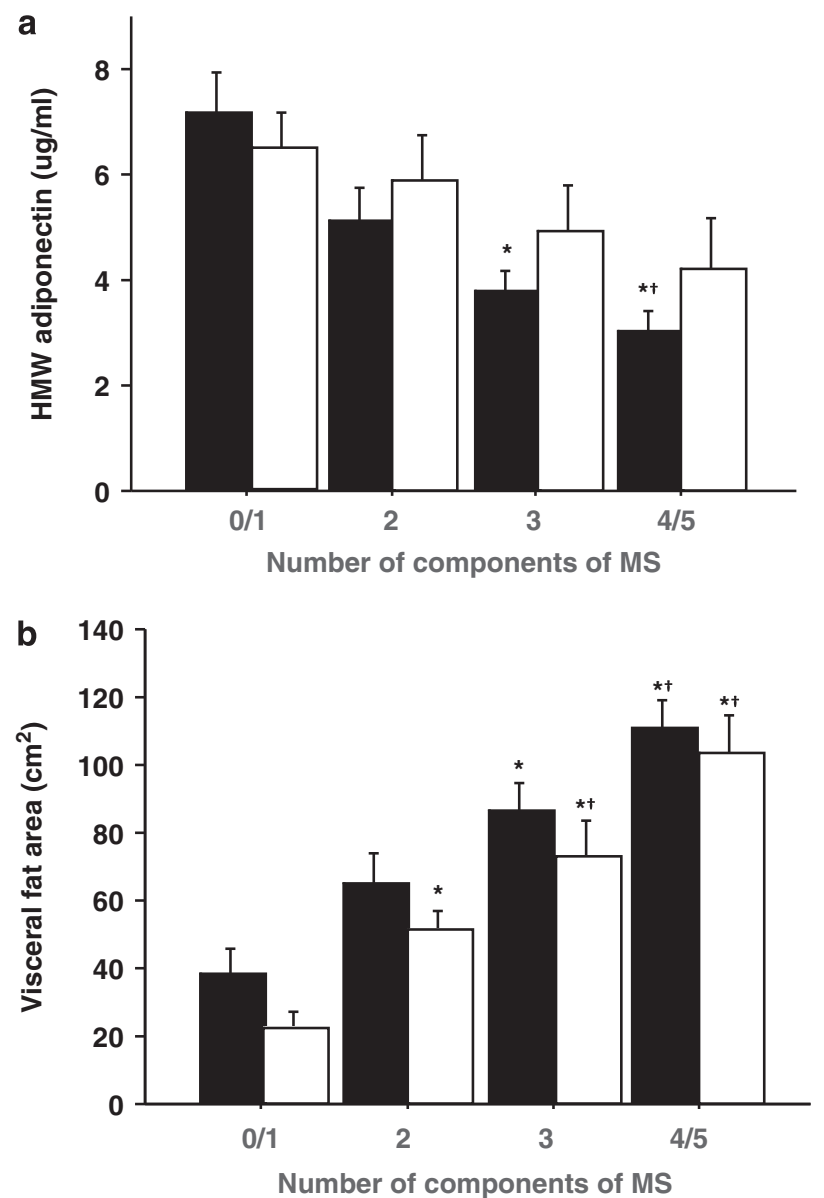

Figure 2 The mean values of high-molecular-weight (HMW) adiponectin (a) and visceral fat area (VFA) (b) according to the number of metabolic syndrome components in male (filled bars) and female (open bars) hemodialysis patients. The data were analyzed using one-way analysis of variance (ANOVA); post hoc comparisons were made using Tukey's honest significance difference test. The bar graphs represent mean \pm s.e.m. ( ${ }^{*} P<0.05$ vs. $0 / 1$ component and ${ }^{\dagger} P<0.05$ vs. 2 components). There was a stepwise decrease in HMW adiponectin levels and increase in VFA corresponding to the accumulation of components of metabolic syndrome (MS) $(P<0.0001$ for trend).

suggesting that the type of medication rather than BP level affected adiponectin levels.

\section{DISCUSSION}

In this study, we observed two specific new findings. First, we found an association between plasma HMW adiponectin levels and the visceral fat obesity determined by direct measurement of VFA using abdominal computed tomography in hemodialysis patients. Second, we found an association of plasma HMW adiponectin levels and HMW-to-total adiponectin ratio with the use of RAS inhibitors and CCBs in hemodialysis patients.

Several investigators have reported that total adiponectin levels are higher in patients on hemodialysis compared with healthy volunteers ${ }^{6,12-14}$ and those with pre-hemodialysis CKD. ${ }^{15}$ In this study, HMW adiponectin levels were significantly higher in hemodialysis patients compared with patients with CKD in both men and women. HMW adiponectin levels of patients with CKD and hemodialysis were higher than those of healthy volunteers as reported previously, ${ }^{9}$ suggesting that impaired renal function may affect the elimination
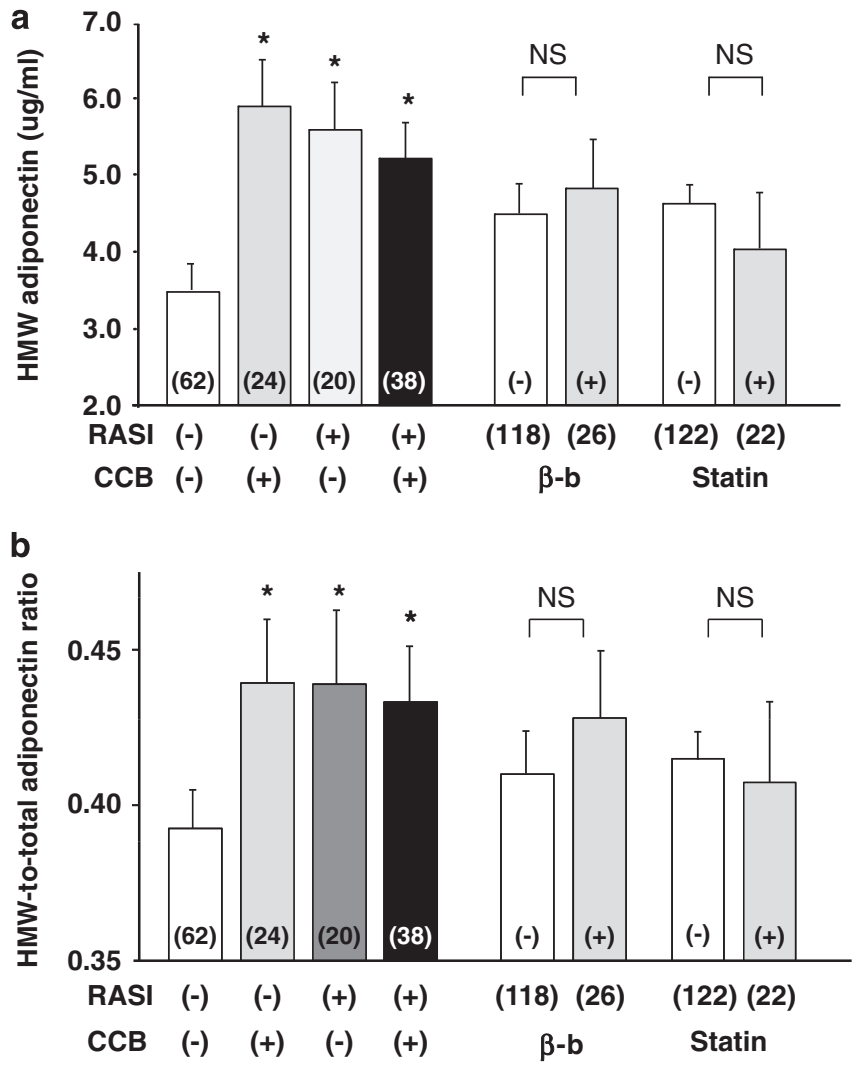

Figure 3 The adjusted mean values of high-molecular-weight (HMW) adiponectin (a) and HMW-to-total adiponectin (b) according to treatment in hemodialysis patients. Adjusted for age, sex, systolic blood pressure (BP), body mass index (BMI), hemoglobin, albumin, previous cardiovascular disease (CVD), smoking, medication for hypertension, medication for diabetes and medication for dyslipidemia. The bar graphs represent mean \pm s.e.m. ( ${ }^{*} P<0.05$ ). RASI, renin-angiotensin system inhibitors; CCB, calcium channel blockers; $\beta$-b, $\beta$-blockers.

of total and HMW adiponectin. Zoccali et al. ${ }^{6}$ suggested that the downregulation of adiponectin receptors may reset serum adiponectin concentrations to a higher level. Recently, Shen et al. ${ }^{12}$ reported that both AdipoR1 and R2 upregulated peripheral blood mononuclear cells of hemodialysis patients in a manner unrelated to insulin resistance, suggesting that adiponectin signaling is an adaptive, protective mechanism in uremia rather than a cause of dysmetabolism. However, it is clear that hemodialysis patients have a high risk of atherosclerotic disease, despite hyperadiponectinemia. As for the reason for this discrepancy, renal impairment might contribute to the decreasing HMW-to-total adiponectin ratio through decreasing HMW adiponectin. Odamaki et al. ${ }^{16}$ reported that VFA is a major determinant of plasma total adiponectin in hemodialysis patients; however, none of the studies have investigated HMW adiponectin levels and directly measured VFA. Thus, we evaluated the correlation between HMW adiponectin and VFA in hemodialysis patients.

We found that plasma HMW adiponectin was negatively associated with VFA in both men and women, but there was no association with BMI and WC in women, suggesting that VFA is a more sensitive determinant of metabolic abnormality than BMI and WC. Women had significantly greater levels of HMW adiponectin than men, which is similar to what has been reported in the general population. ${ }^{4,5,17}$ Thus, to minimize the influence of age and gender, we performed a multivariate analysis adjusted for age, gender, duration of dialysis and 
Table 5 Characteristics of patients treated with RAS inhibitors or CCBs in hemodialysis patients

\begin{tabular}{|c|c|c|c|c|}
\hline RAS inhibitors & $(-)$ & $(-)$ & $(+)$ & $(+)$ \\
\hline CCBs & $(-)$ & $(+)$ & $(-)$ & $(+)$ \\
\hline Number & 65 & 23 & 18 & 38 \\
\hline Sex, men (\%) & 66 & 56 & 61 & 61 \\
\hline Duration of dialysis (months) & $138.8 \pm 105.3$ & $94.5 \pm 89.8$ & $129.4 \pm 111.2$ & $140.6 \pm 111.9$ \\
\hline Waist circumference $(\mathrm{cm})$ & $83.3 \pm 9.4$ & $82.4 \pm 9.0$ & $83.1 \pm 11.3$ & $84.3 \pm 10.1$ \\
\hline Systolic BP (mm Hg) & $139.4 \pm 22.2$ & $146.7 \pm 24.3$ & $150.1 \pm 24.7$ & $152.8 \pm 20.4$ \\
\hline Diastolic BP $(\mathrm{mm} \mathrm{Hg})$ & $78.7 \pm 12.5$ & $82.3 \pm 13.6$ & $84.7 \pm 14.1$ & $84.9 \pm 12.8$ \\
\hline Pulse pressure $(\mathrm{mm} \mathrm{Hg})$ & $59.8 \pm 17.7$ & $64.3 \pm 15.9$ & $65.4 \pm 17.8$ & $67.9 \pm 17.1$ \\
\hline
\end{tabular}

Abbreviations: BP, blood pressure; CCB, calcium channel blocker; RAS, renin-angiotensin system.

other conventional risk factors, and showed that the independent contribution of VFA to decreased plasma HMW adiponectin remained significant in hemodialysis patients. Furthermore, we showed that the distribution of abdominal adiposity and the prevalence of VFA $>100 \mathrm{~cm}^{2}$ was $31.2 \%$ in men and $15.5 \%$ in women and that the accumulation of MS components was significant and correlated positively with VFA and negatively with HMW adiponectin, suggesting that reduced VFA might be important for the prevention of CVD not only in the general population, but also in hemodialysis patients.

In contrast to the relationships found in the general population, numerous studies have found that adiposity has a neutral or even protective association with mortality in hemodialysis patients, possibly due to the so-called reverse epidemiology. ${ }^{18-20}$ However, there is the possibility that the protective link observed between obesity and mortality may not actually exist. Most studies have not completely accounted for known mortality risk factors such as smoking, BP and medication. The incidence of cardiovascular disease is high even in non-obese individuals with a BMI within the normal range who have an accumulation of visceral fat. ${ }^{21}$ Recently, VFA has been significantly associated with insulin resistance ${ }^{22}$ and with the prevalence of CVD in participants from the Framingham Heart Study; ${ }^{23}$ thus, the accurate assessment of both body fat distribution and VFA is critical for assessing the risk of arteriosclerotic disease. Ohkawa et al. ${ }^{24}$ reported that VFA in hemodialysis patients increased irrespective of BMI, and a positive relationship existed between VFA and atherosclerosis. Thus, visceral fat obesity is not rare in Japanese hemodialysis patients, and this shift of abdominal adiposity might be associated with increased risks of CVD in hemodialysis patients.

Finally, we showed here for the first time that levels of HMW adiponectin and HMW-to-total adiponectin ratio in patients taking RAS inhibitors, CCBs and a combination of RAS inhibitors and CCBs were significantly higher than that in patients not on these treatments after adjustment for age, sex, BMI and other cardiovascular risk factors. It has been reported that RAS inhibitors ${ }^{25,26}$ and $\mathrm{CCBs}^{27}$ increase adiponectin levels in non-hemodialysis patients. Previous studies have shown that angiotensin II receptor blocker inhibits obesity-induced hypoadiponectinemia through the inhibition of reactive oxygen species generation in mice ${ }^{28}$ and that some angiotensin II receptor blockers have peroxisome proliferator-activated receptor $\gamma$-activating properties, ${ }^{29}$ thereby stimulating adiponectin production. Some CCBs have also been reported to have the ability to increase adiponectin levels through the inhibition of reactive oxygen species generation ${ }^{30}$ and of monocyte and platelet activation. ${ }^{27}$ On the basis of these reports, we speculated that the blockade of RAS and of the calcium channel may be a powerful tool not only in patients with hypertension and pre-hemodialysis CKD, but also in hemodialysis patients for improving metabolic disturbance. In contrast, some studies have reported that statins increased circulating levels of adiponectin. ${ }^{31,32}$ In this study, we found no significant differences between patients treated with or without statins.

The limitations of the study were, first, that the definition of MS is still under debate. In Japan, a WC of $85 \mathrm{~cm}$ for men and $90 \mathrm{~cm}$ for women has been adopted as the definition of MS, which approximates a VFA of $100 \mathrm{~cm}^{2}$ at the level of the umbilicus and is indicative of the risk of MS. ${ }^{11}$ Thus, we used VFA $>100 \mathrm{~cm}^{2}$ as the cutoff value for visceral fat obesity. Second, we used non-fasting data, in particular, non-fasting high serum triglycerides and low HDL cholesterol, as a component of MS. Although justification by the same cutoff point during fasting is still under debate, the data of non-fasting triglycerides can be used because it is a significant predictor for ischemic heart disease. ${ }^{33}$ Therefore, we assessed $\mathrm{HbA}_{1 \mathrm{c}}$ instead of fasting glucose, non-fasting triglycerides and HDL cholesterol and used a 'modified' MS definition rather than the standard definition. Third, we could not compare the adiponectin levels before and after medication in the present cross-sectional, observational study of chronic hemodialysis patients. However, there were no significant differences in WC, VFA and subcutaneous fat area among patient groups treated with or without RAS inhibitors/CCBs, suggesting that types of medications rather than abdominal obesity affected the adiponectin levels. The effect of RAS inhibitors and CCBs on adiponectin levels is still under debate. Huang et al. ${ }^{20}$ also investigated the effect of the RAS blockade and did not find any effect on adiponectin levels. However, several investigators reported that angiotensin-converting enzyme inhibitor, angiotensin II receptor blocker ${ }^{25,26}$ and $\mathrm{CCB}^{27}$ increase adiponectin levels in non-hemodialysis patients. Thus, we speculated that these drugs might also increase adiponectin levels in hemodialysis patients. Fourth, the Japanese hemodialysis population is clearly different from others, such as that of the United States, which has a higher proportion of diabetic patients and African Americans. ${ }^{34}$ There are several other differences in terms of patient age and sex distribution; therefore, the results may not be directly applicable to westerners or individuals of certain ethnic groups. Thus, we must consider the potential associations between clinical practice and outcomes, adjusted for patient characteristics, which may affect clinical care. Furthermore, the relations to cardiovascular end points must be examined in a future large prospective study. 
In conclusion, we showed that plasma HMW adiponectin levels were associated negatively with VFA and that the blockade of RAS as well as of the calcium channel might be effective for improving adipocytokine-related metabolic abnormalities in hemodialysis patients. These results highlight the importance of modern therapeutic efforts aimed at ameliorating metabolic disturbance in hemodialysis patients.

\section{CONFLICT OF INTEREST}

The authors declare no conflict of interest.

1 Wahba IM, Mak RH. Obesity and obesity-initiated metabolic syndrome: mechanistic links to chronic kidney disease. Clin J Am Soc Nephrol 2007; 2: 550-562.

2 Parfrey PS, Foley RN. The clinical epidemiology of cardiac disease in chronic renal failure. J Am Soc Nephrol 1999; 10: 1606-1615.

3 Chandran M, Phillips SA, Ciaraldi T, Henry RR. Adiponectin: more than just another fat cell hormone? Diabetes Care 2003; 26: 2442-2450.

4 Matsuzawa Y, Funahashi T, Kihara S, Shimomura I. Adiponectin and metabolic syndrome. Arterioscler Thromb Vasc Biol 2004; 24: 29-33.

5 Kumada M, Kihara S, Sumitsuji S, Kawamoto T, Matsumoto S, Ouchi N, Arita Y, Okamoto Y, Shimomura I, Hiraoka H, Nakamura T, Funahashi T, Matsuzawa Y, Osaka CADSG. Association of hypoadiponectinemia with coronary artery disease in men. Arterioscler Thromb Vasc Biol 2003; 23: 85-89.

6 Zoccali C, Mallamaci F, Tripepi G, Benedetto FA, Cutrupi S, Parlongo S, Malatino LS, Bonanno G, Seminara G, Rapisarda F, Fatuzzo P, Buemi M, Nicocia G, Tanaka S, Ouchi $\mathrm{N}$, Kihara S, Funahashi T, Matsuzawa Y. Adiponectin, metabolic risk factors, and cardiovascular events among patients with end-stage renal disease. J Am Soc Nephrol 2002; 13: 134-141.

7 Kadowaki T, Yamauchi T. Adiponectin and adiponectin receptors. Endocr Rev 2005; 26: 439-451.

8 Daugirdas JT. 2nd-generation logarithmic estimates of single-pool variable volume Kt/ V—an analysis of error. J Am Soc Nephrol 1993; 4: 1205-1213.

9 Ebinuma H, Miyazaki O, Yago H, Hara K, Yamauchi T, Kadowaki T. A novel ELISA system for selective measurement of human adiponectin multimers by using proteases. Clin Chim Acta 2006; 372: 47-53.

10 Grundy SM, Cleeman JI, Merz CNB, Brewer HB, Clark LT, Hunninghake DB, Pasternak RC, Smith SC, Stone NJ. Implications of recent clinical trials for the National Cholesterol Education Program Adult Treatment Panel III guidelines. Circulation 2004; 110: 227-239.

11 Matsuzawa Y, Nakamura T, Takahashi M, Ryo M, Inoue S, Ikeda Y, Ohno M, Sakata T, Fukagawa K, Saitoh Y, Sato Y, Shirai K, Miyazaki S, Tokunaga K, Yamanouchi K, Shibasaki T, Nagai M. New criteria for 'obesity disease' in Japan. Circ J 2002; 66: 987-992.

12 Shen YY, Charlesworth JA, Kelly JJ, Loi KW, Peake PW. Up-regulation of adiponectin, its isoforms and receptors in end-stage kidney disease. Nephrol Dial Transp/ 2007; 22: 171-178.

13 Shoji T, Shinohara K, Hatsuda S, Kimoto E, Fukumoto S, Emoto M, Tahara H, Koyama $\mathrm{H}$, Ishimura E, Miki T, Tabata T, Nishizawa Y. Altered relationship between body fat and plasma adiponectin in end-stage renal disease. Metabolism 2005; 54: 330-334.

14 Huang JW, Yen CJ, Chiang HW, Hung KY, Tsai TJ, Wu KD. Adiponectin in peritoneal dialysis patients: a comparison with hemodialysis patients and subjects with normal renal function. Am J Kidney Dis 2004; 43: 1047-1055.

15 Komaba H, Igaki N, Goto S, Yokota K, Doi H, Takemoto T, Kohno M, Hirosue Y, Goto T. Increased serum high-molecular-weight complex of adiponectin in type 2 diabetic patients with impaired renal function. Am J Nephrol 2006; 26: 476-482.

16 Odamaki M, Furuya R, Kinumura $\mathrm{Y}$, Ikegaya $\mathrm{N}$, Kumagai $\mathrm{H}$. Association between plasma adiponectin concentration and visceral fat accumulation in hemodialysis patients. Nephron Clin Pract 2006; 102: C8-C13.
17 Adamczak M, Rzepka E, Chudek J, Wiecek A. Ageing and plasma adiponectin concentration in apparently healthy males and females. Clin Endocrinol 2005; 62: 114-118.

18 Kopple JD, Zhu XF, Lew NL, Lowrie EG. Body weight-for-height relationships predict mortality in maintenance hemodialysis patients. Kidney Int 1999; 56: 1136-1148.

19 Kalantar-Zadeh K, Block G, Humphreys MH, Kopple JD. Reverse epidemiology of cardiovascular risk factors in maintenance dialysis patients. Kidney Int 2003; 63 : 793-808.

20 Stenvinkel P, Carrero JJ, Axelsson J, Lindholm B, Heimbuerger O, Massy Z. Emerging biomarkers for evaluating cardiovascular risk in the chronic kidney disease patient: how do new pieces fit into the uremic puzzle? Clin J Am Soc Nephrol 2008; 3: 505-521.

21 Nakamura T, Tokunaga K, Shimomura I, Nishida M, Yoshida S, Kotani K, Islam A, Keno Y, Kobatake T, Nagai Y, Fujioka S, Tarui S, Matsuzawa Y. Contribution of visceral fat accumulation to the development of coronary-artery disease in nonobese men. Atherosclerosis 1994; 107: 239-246.

22 Gohda T, Gotoh H, Tanimoto M, Sato M, lo H, Kaneko K, Hamada C, Tomino Y. Relationship between abdominal fat accumulation and insulin resistance in hemodialysis patients. Hypertens Res 2008; 31: 83-88.

23 Mahabadi AA, Massaro JM, Rosito GA, Levy D, Murabito JM, Wolf PA, O'Donnell CJ, Fox CS, Hoffmann U. Association of pericardial fat, intrathoracic fat, and visceral abdominal fat with cardiovascular disease burden: the Framingham Heart Study. Eur Heart J 2009; 30: 850-856.

24 Ohkawa S, Odamaki M, Ikegaya N, Hibi I, Miyaji K, Kumagai H. Association of age with muscle mass, fat mass and fat distribution in non-diabetic haemodialysis patients. Nephrol Dial Transpl 2005; 20: 945-951.

25 Furuhashi M, Ura N, Higashiura K, Murakami H, Tanaka M, Moniwa N, Yoshida D, Shimamoto K. Blockade of the renin-angiotensin system increases adiponectin concentrations in patients with essential hypertension. Hypertension 2003; 42: $76-81$.

26 Koh KK, Quon MJ, Han SH, Chung WJ, Kim JA, Shin EK. Vascular and metabolic effects of candesartan: insights from therapeutic interventions. J Hypertens 2006; 24: S31-S38.

27 Nomura S, Inami N, Kimura Y, Omoto S, Shouzu A, Nishikawa M, Iwasaka T. Effect of nifedipine on adiponectin in hypertensive patients with type 2 diabetes mellitus. J Hum Hypertens 2007; 21: 38-44.

28 Kurata A, Nishizawa $H$, Kihara $S$, Maeda $N$, Sonoda M, Okada $T$, Ohashi $K$, Hibuse T, Fujita K, Yasui A, Hiuge A, Kumada M, Kuriyama H, Shimomura I, Funahashi T. Blockade of angiotensin II type-1 receptor reduces oxidative stress in adipose tissue and ameliorates adipocytokine dysregulation. Kidney Int 2006; 70: 1717-1724.

29 Clasen R, Schupp M, Foryst-Ludwig A, Sprang C, Clemenz M, Krikov M, Thone-Reineke $\mathrm{C}$, Unger T, Kintscher U. PPAR gamma-activating angiotensin type-1 receptor blockers induce adiponectin. Hypertension 2005; 46: 137-143.

30 Yamagishi S, Inagaki Y, Nakamura K, Imaizumi T. Azelnidipine a newly developed longacting calcium antagonist, inhibits tumor necrosis factor-alpha-induced interleukin-8 expression in endothelial cells through its anti-oxidative properties. J Cardiovasc Pharmacol 2004; 43: 724-730.

31 Nakamura T, Kodama Y, Takano H, Umetani K, Fujioka D, Saito Y, Kawabata KI, Obata JE, Kitta Y, Kobayashi T, Mende A, Kugiyama K. Increase in circulating levels of adiponectin after treatment with statin and fibrate in patients with coronary artery disease and hyperlipidemia. Atherosclerosis 2007; 193: 449-451.

32 Saito S, Fujiwara T, Matsunaga T, Minagawa K, Fukui K, Fukuda I, Osanai T, Okumura $\mathrm{K}$. Increased adiponectin synthesis in the visceral adipose tissue in men with coronary artery disease treated with pravastatin: a role of the attenuation of oxidative stress. Atherosclerosis 2008; 199: 378-383.

33 McNeill AM, Schmidt MI, Rosamond WD, East HE, Girman CJ, Ballantyne CM, Golden $\mathrm{SH}$, Heiss G. The metabolic syndrome and 11-year risk of incident cardiovascular disease in the atherosclerosis risk in communities study. Diabetes Care 2005; 28 : 385-390.

34 Young EW, Goodkin DA, Mapes DL, Port FK, Keen ML, Chen K, Maroni BL, Wolfe RA, Held PJ. The dialysis outcomes and practice patterns study (DOPPS): an international hemodialysis study. Kidney Int 2000; 57: S74-S81. 